\title{
Immersive Video Modeling Versus Traditional Video Modeling for Teaching Central Venous Catheter Insertion to Medical Residents
}

\author{
Evan Mah ${ }^{1,2}$, Julie Yu ${ }^{3}$, Megan Deck ${ }^{4}$, Kish Lyster $^{5}$, Joann Kawchuk ${ }^{3}$, Alison Turnquist ${ }^{6}$, Brent Thoma ${ }^{6}$ \\ 1. Department of Family Medicine, University of British Columbia, Campbell River, CAN 2. College of Medicine, \\ University of Saskatchewan, Saskatoon, CAN 3. Department of Anesthesiology, Perioperative Medicine, and Pain \\ Management, University of Saskatchewan, Saskatoon, CAN 4. Department of Anesthesiology, University of \\ Saskatchewan, Saskatoon, CAN 5. Department of Family Medicine, University of Saskatchewan, Regina, CAN 6. \\ Department of Emergency Medicine, University of Saskatchewan, Saskatoon, CAN
}

Corresponding author: Brent Thoma, thoma.brent@gmail.com

\section{Abstract}

\section{Background}

Central Venous Catheter (CVC) placement is a common critical care procedure. Simulated practice has been shown to reduce its iatrogenic complications. Video modeling (VM) is an instructional adjunct that improves the quality and success of CVC insertion. Immersive VM can improve recall and skill translation, but its role in teaching medical procedures is not established.

\section{Research question/hypothesis}

We hypothesized that, relative to traditional VM, immersive VM would decrease cognitive load and enhance ultrasound-guided CVC insertion skill acquisition.

\section{Methods}

Thirty-two resident physicians from four specialties were randomized into traditional (control) or immersive VM (intervention) groups for three CVC training sessions. Cognitive load was quantified via NASA Task Load Index (TLX). Mean ( \pm standard deviations) values were compared using two-tailed t-tests. Skill acquisition was quantified by procedural time and the average 5-point [EM1] [TB2] entrustment score of three expert raters.

\section{Results}

Overall entrustment scores improved from the first $(3.44 \pm 0.98)$ to the third $(4.06 \pm 1.23 ; \mathrm{p}<0.002)$ session but were not significantly different between the control and intervention groups. There were no significant differences between NASA TLX scores or procedural time.

Review began 01/25/2021 Review ended 02/23/2021 Published 03/02/2021

\section{() Copyright 2021}

Mah et al. This is an open access article distributed under the terms of the Creative Commons Attribution License CC-BY 4.0., which permits unrestricted use, distribution, and reproduction in any medium, provided the original author and source are credited.

\section{Conclusion}

We found no significant difference in entrustment, cognitive load, or procedural time. Immersive VM was not found to be superior to traditional VM for teaching CVC insertion.

Categories: Emergency Medicine, Internal Medicine, Medical Education

Keywords: simulation in medical education, simulation design, video-based learning, 360-degree video recording, medical resident education, video modeling, central venous catheter, skills and simulation training

\section{Introduction}

Central venous catheter (CVC) placement is a medical procedure that involves the insertion of a catheter into the venous system to facilitate the administration of medications, fluids, and blood products [1]. It has been associated with serious mechanical (pneumothorax, bleeding, thrombus formation, occlusion, extravasation, catheter embolism or breakage, fistula formation, air embolism, pericardial tamponade, cardiac aneurysm, or vein stenosis) and infectious (cellulitis, phlebitis, intracardiac abscess, or sepsis) complications. These complications vary in prevalence due to different definitions, reporting patterns, site selection, the catheter used, dressing standards, patient choice, and provider experience [2].

Expert video modeling is a common component of medical simulation training that originated in athletics training [3]. It allows trainees to witness the performance of a procedure to develop self-efficacy and confidence [4]. CVC insertion training that utilizes both video modeling (VM) and task trainers for procedural simulation has been shown to decrease instructor time [5,6], training time [5], equipment spoilage [6], and adverse events [5,7]. The merit of traditional VM has been demonstrated in procedural 


\section{Cureus}

skills training, particularly in sports $[3,8,9]$. Recently, more immersive educational technologies, such as virtual reality headsets, have been made available at a reasonable cost, but their application has not been explored in this context.

Immersive VM uses video capture technology and a virtual reality headset that surrounds the user with a convincing replica of the same environment that they might expect in a real-life setting [10]. Immersive VM has been demonstrated to promote learner engagement in the tasks they observe [11-14]. This increased engagement could conceivably improve retention and learning. Additionally, immersive VM has a complicated impact on cognitive load. Frederiksen et al. found that performing procedures in immersive virtual reality increases cognitive load [15]. However, cognitive load decreased with repeated exposure. They hypothesized that through repeated exposure to higher cognitive load during training, trainees may be better prepared for complex real-world performance [15].

Building on this literature, we hypothesized that immersive VM would be acceptable to learners and that, relative to traditional VM, immersive VM would result in enhanced ultrasound-guided CVC insertion skill acquisition mediated by the decreased cognitive load during procedure performance.

This research was previously presented at the University of Saskatchewan Medical Education Research and Scholarship Day (June 8, 2018), the College of Medicine Fall Poster Day (November 23, 2018), and the Canadian Conference on Medical Education (April 14, 2019).

\section{Materials And Methods}

The University of Saskatchewan Research Ethics Board deemed this study exempt from ethical review by (BIO\# 18-46). The Regina Qu'Appelle Health Region provided operational approval.

All 32 first-year residents from four training programs (anesthesia, emergency medicine, general surgery, and internal medicine) in Saskatoon and Regina (Saskatchewan) were enrolled in a CVC insertion training program at our institution and were invited to participate in the study. Consent was provided by each participant prior to the first session and demographic information was collected (Appendix A).

Expert instructional procedural videos were created simultaneously in traditional (Video 1) and immersive (Video 2) video formats. The traditional format was a fixed, two-dimensional video viewed on the screen of a Samsung S6 32GB cell phone (Samsung Electronics, Suwon, South Korea). The immersive format was a video of a 180-degree wide field of view that was viewed on a Samsung S6 32GB cell phone placed in a Samsung Gear Virtual Reality Headset (2016 edition, Samsung Electronics). The headset allowed the user to raise, lower, and turn their head to change their visual focus and prevented visual input from their true surroundings. We recorded the instructional procedural videos using a CVC Insertion Kit (Teleflex, Wayne, USA) and a CVC Internal Internal Jugular Task Trainer (Simulab, Seattle, USA) with a Samsung S6 32GB cell phone (Video 1) and a 360fly 4K Video Camera (360fly, Canonsburg, USA) (Video 2). Both videos were edited with the same instructive audio narrative using Premiere Pro (Adobe, Inc., San Jose, USA). Equipment costs are outlined in Appendix B.

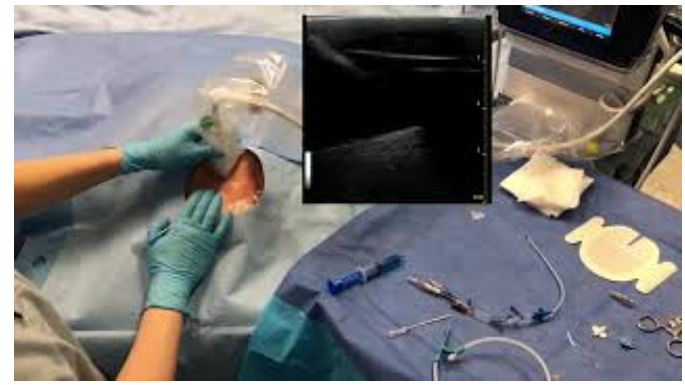

VIDEO 1: Traditional Video Model

View video here: https://www.youtube.com/watch?v=0-nITvvVC5M 


\section{Cureus}

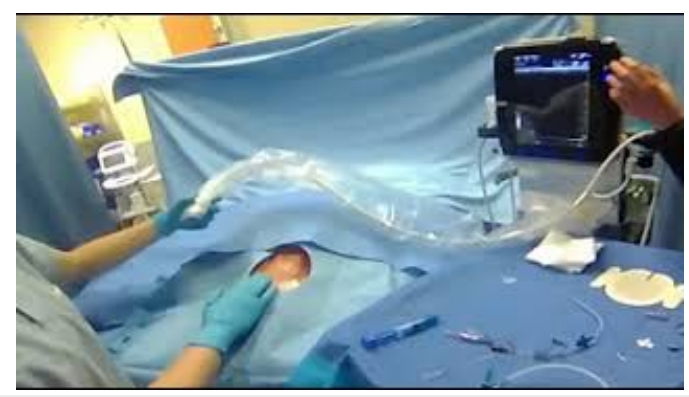

\section{VIDEO 2: 360 Video Model v2.0}

View video here: https://www.youtube.com/watch?v=QGvJfbNJg6c

The ultrasound-guided, CVC insertion training program spanned three three-hour sessions over a 12-week period. Each group contained three to four residents. All residents were provided with prereading material outlining the procedure. At the beginning of the first session, all residents received procedural instruction by a staff physician experienced in CVC insertion. The instructor taught a standardized, step-wise approach to CVC insertion based upon our institution's best practices. The resident groups were randomized to the control (traditional VM) or the interventional (immersive VM) groups. We aimed to maintain the composition of each instructional group throughout the study, but in cases where this was not possible because the residents changed instructional groups, they conducted their VM consistent with the study group that they were initially assigned. Following the viewing, both groups practiced the skill on task trainers. Individualized feedback was provided concurrently by the instructors throughout the practice session. The subsequent two training sessions followed the same video review and practice format but did not include dedicated instruction at the beginning. Concurrent feedback was provided by instructors through all of the sessions.

At the end of each of the three training sessions, each participant completed a one-on-one testing session supervised by a staff physician instructor. These sessions were video recorded to include the procedural area, the participant's hands and arms, and the ultrasound screen. No feedback was provided during this test session. After each session, participants were asked to complete a NASA Task Load Index (TLX), a multidimensional scoring system that assesses cognitive workload (Appendix C), while the observing instructor completed an assessment form consisting of the O-Score entrustment scale [16] and narrative feedback. Participants were stopped by the instructor if they exceeded 15-minutes of procedural time. Time zero began with the first needle insertion into the skin and concluded with the placement of the Tegaderm ${ }^{\mathrm{TM}}$ (3M Company, Siant Paul, USA) dressing to secure the CVC. Task success was defined as the completion of the task within the time frame. Failure was defined as the failure to complete the procedure within the allotted time.

Following each participants' testing session, their procedural and ultrasound videos were combined into a single video (Figure 1). These videos were saved on a password-protected hard drive with file names based on a computerized random number order generator from 100-400. Time from first needle insertion to Tegaderm $^{\mathrm{TM}}$ placement was determined by a blinded study investigator. Two additional staff physician investigators, who were not involved in the participant's recorded teaching session but were familiar with the curriculum and its assessment, performed blinded assessments of each recorded CVC insertion using the O-Score entrustment scale completed via SurveyMonkey (SVML Inc., San Mateo, USA) (Appendix D).

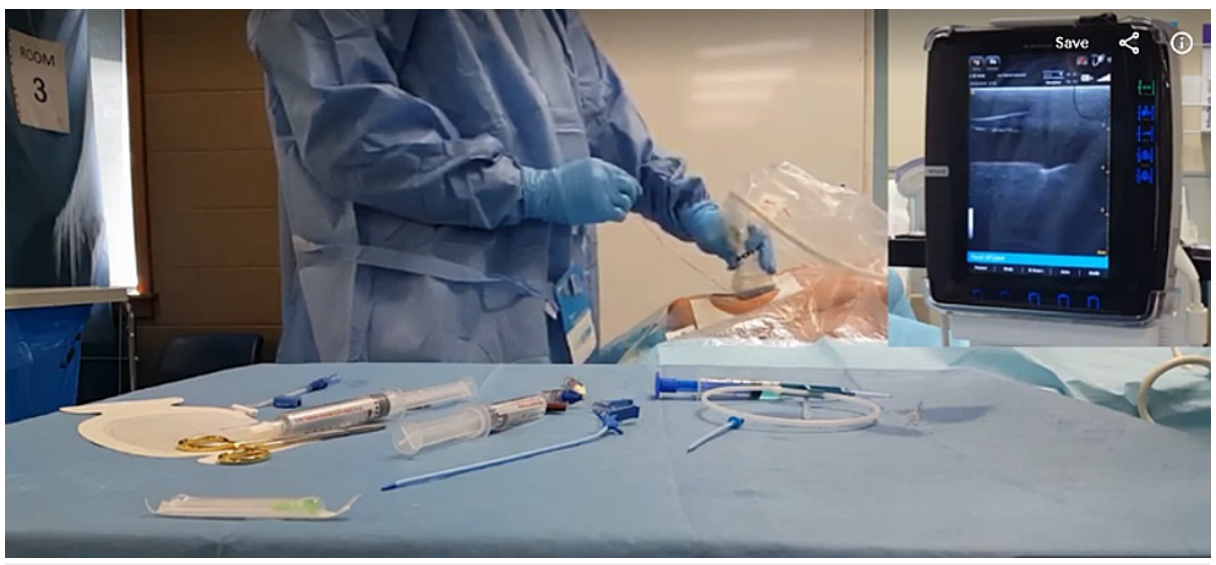

FIGURE 1: A sample of the video created from a participant's test session, showing their performance with the video of the ultrasound 
Procedural competence was assessed using the average of the three entrustment scores (one bedside rater and two video raters) and procedure length (from first needle insertion to Tegaderm ${ }^{\mathrm{TM}}$ placement). Cognitive load during simulated CVC placement was assessed using the NASA TLX score completed by each participant following each test session. These variables were compared between sessions within groups, as well as between groups using paired and unpaired two-tailed t-tests as appropriate. A power calculation conducted using an alpha of 0.05 and a beta of 0.8 seeking a one-point difference on the entrustment score between the groups required two groups of 16 participants.

Lastly, the acceptability of immersive and traditional VM was explored by comparing CVC insertion training program evaluations from residents in each group. The program was evaluated using a modified version of the evaluation of technology-enhanced learning materials learner perspective (ETELM-LP) survey [17]. Participants completed the survey online using SurveyMonkey (Appendix E) after all three sessions were completed and the results were compared by group using t-tests.

\section{Results}

All 32 first-year eligible for the study participated. Five additional third-year residents participated in the training program but were not eligible for the study. Group demographics are outlined in Table 1. All study participants were right-handed. Most participants ( $\mathrm{n}=19$ or $59.4 \%$ ) were internal medicine residents. Nine participants (28.1\%) had received formal instruction on CVC insertion prior to the training program. The control and intervention groups differed in size because the residents were randomized by group and the number of eligible residents in each group varied.

\begin{tabular}{|c|c|c|c|c|c|}
\hline & \multirow{2}{*}{ Male } & \multirow{2}{*}{ Female } & \multirow{2}{*}{ Previous training } & \multicolumn{2}{|c|}{ Reviewed course material prior to the first session } \\
\hline & & & & Yes & Partially \\
\hline Control & 13 (72\%) & $5(27.7 \%)$ & $6(33 \%)$ & 10 & 3 \\
\hline Intervention & $10(71 \%)$ & $4(28.6 \%)$ & $3(21 \%)$ & 8 & 6 \\
\hline
\end{tabular}

\section{TABLE 1: Demographic characteristics of the control and intervention groups}

Cognitive load (as assessed by the NASA TLX) and procedural competence (as assessed by procedural time and entrustment score) are reported in Figures 2-4. There was no difference in cognitive load during the procedure in the immersive VM group. Further, there was no change in cognitive load through the three study sessions. There was a significant improvement in entrustment score (mean \pm SD) from the first $(3.4 \pm 1.0)$ to the third $(4.1 \pm 1.2 ; \mathrm{p}<0.001)$ session across all participants. There were no statistical differences in entrustment scores between the control and intervention groups during the last session. Time to procedure completion did not significantly change from the first to the third session in either group.

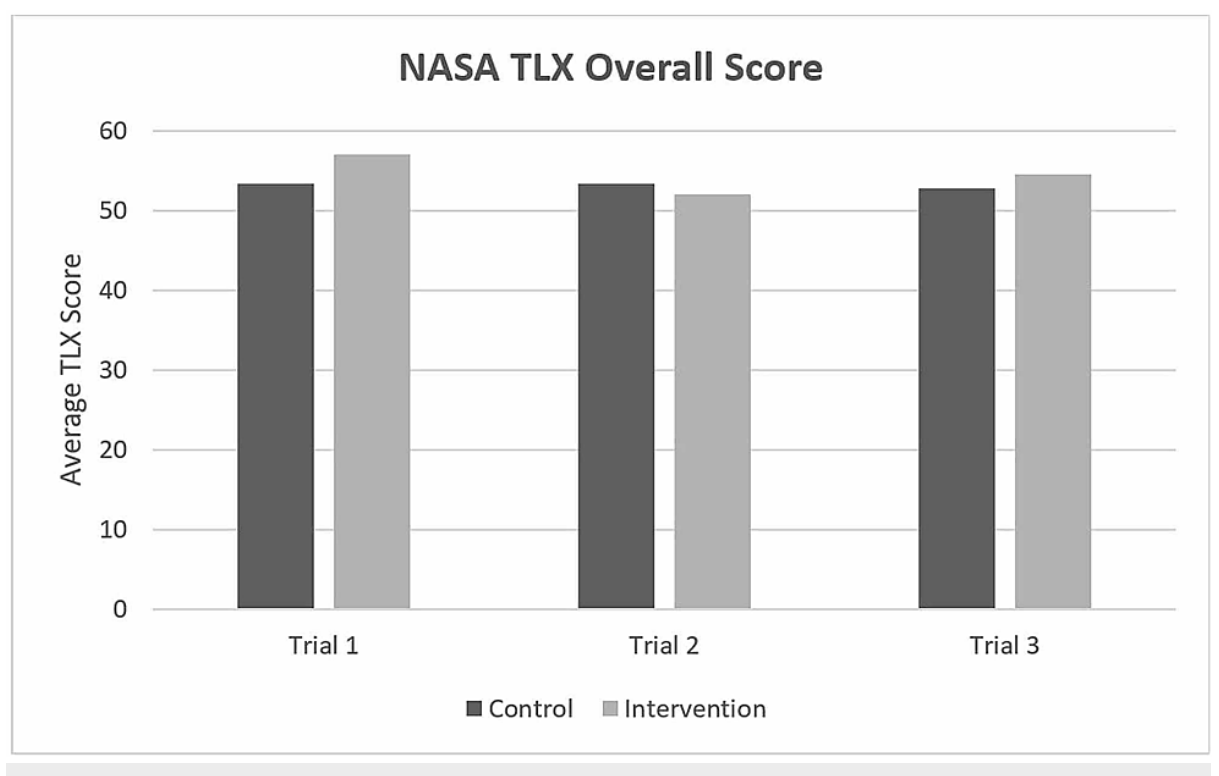




\section{Cureus}

FIGURE 2: A graph of the average NASA TLX scores for both groups over the three trials. There were no statistically significant differences between groups or between testing sessions.

TLX: Task Load Index

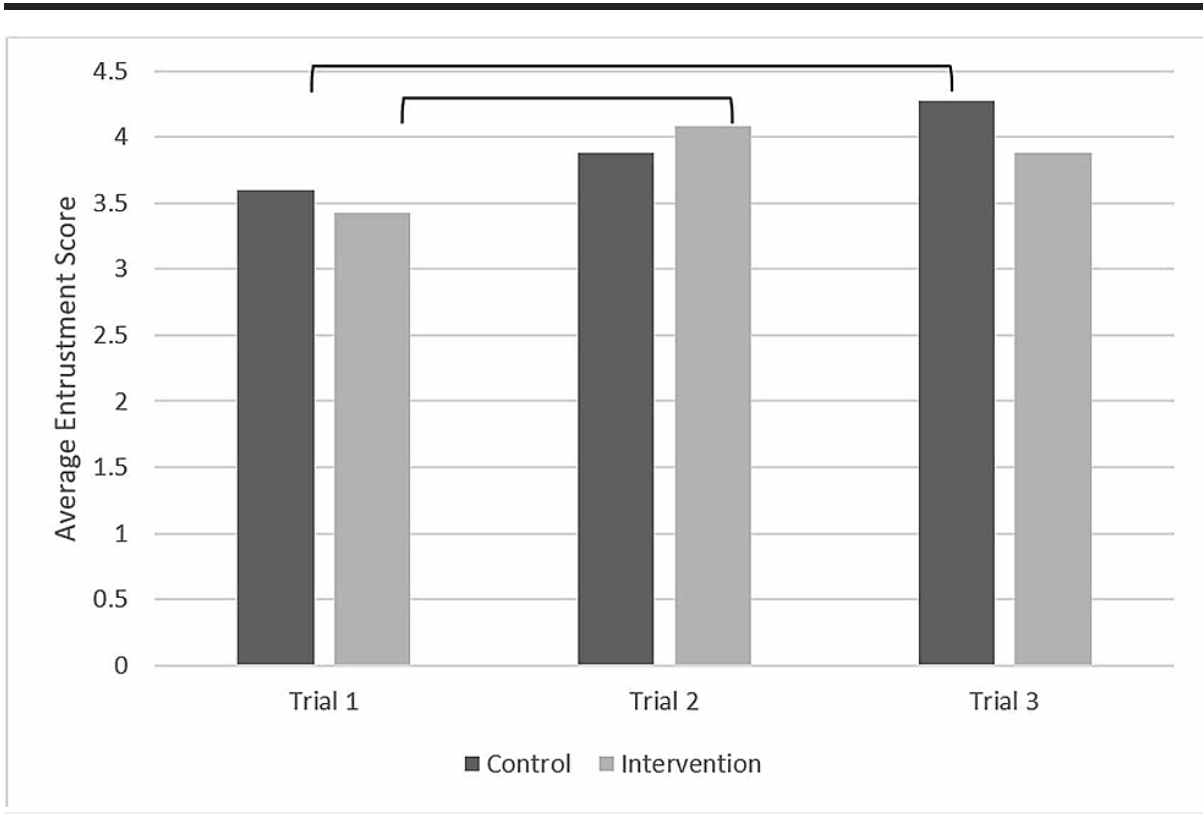

FIGURE 3: Average entrustment scores for both control and intervention groups over the three testing sessions. The bars indicate there is statistically significant difference at $p<0.05$.

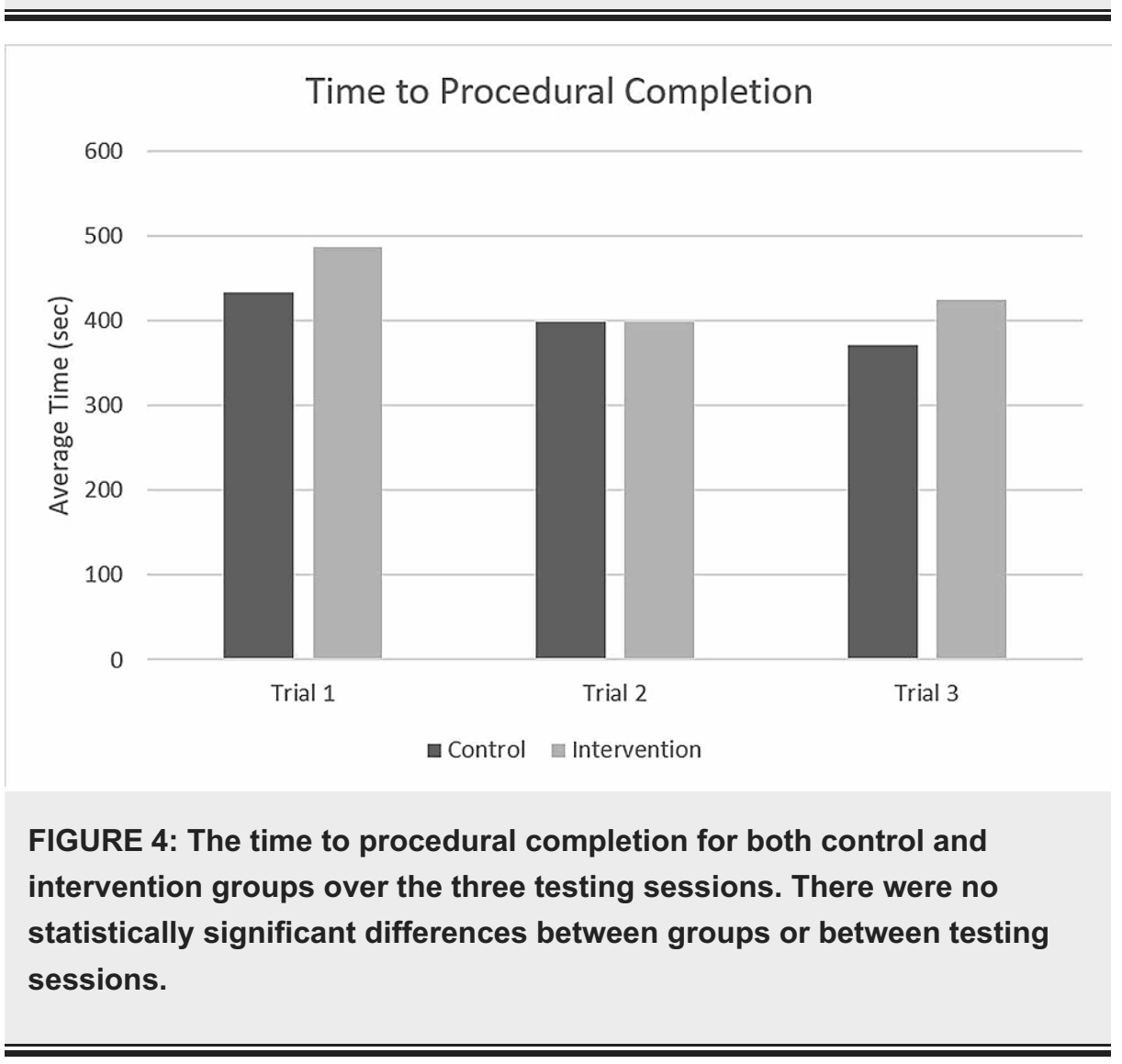


Each item on the ETELM-LP (Appendix F) was rated from strongly disagree (1) to strongly agree (7). Significant differences between the groups are outlined in Table 2. Participants in the control group reported higher agreement that navigation of the technology-based components of the course was logical, consistent, and efficient; that the technology and media supported the learning objectives; that the course did not require inappropriately high technology skills; and that they did not have significant technical problems during the course. The control group also reported higher agreement that the quality of the video review helped them learn the skill, contributed to the achievement of the learning objectives, and was consistent with the instructor's teaching of the pre-course material.

\begin{tabular}{|c|c|c|c|}
\hline ETELM Question & $\begin{array}{l}\text { Control } \\
\text { Average }\end{array}$ & $\begin{array}{l}\text { Intervention } \\
\text { Average }\end{array}$ & $\begin{array}{l}\text { p- } \\
\text { value }\end{array}$ \\
\hline $\begin{array}{l}\text { Navigation of the technology-based components of the course was logical, consistent, } \\
\text { and efficient }\end{array}$ & 6.3 & 5.4 & 0.03 \\
\hline The course technologies and media supported the learning objectives & 6.3 & 5.5 & 0.05 \\
\hline This course did not require inappropriately high technology skills & 6.7 & 5.8 & 0.005 \\
\hline I did not have significant technical problems during this course & 6.5 & 5.3 & 0.02 \\
\hline The quality of the video review of the procedure helped me to learn the skill & 6.4 & 5.5 & 0.03 \\
\hline $\begin{array}{l}\text { The video review of the procedure was consistent with the instructor's teaching and } \\
\text { pre-course material }\end{array}$ & 6.4 & 5.6 & 0.02 \\
\hline $\begin{array}{l}\text { The video review of the procedure contributed to the achievement of course learning } \\
\text { objectives }\end{array}$ & 6.5 & 5.5 & 0.008 \\
\hline
\end{tabular}

TABLE 2: Statistically significant differences from the ETELM Learner Perspective Survey between the control and intervention groups

ETELM: evaluation of technology-enhanced learning materials

\section{Discussion}

Contrary to our hypotheses, immersive VM did not significantly reduce the procedural cognitive load or improve the competence of first-year resident physicians relative to traditional VM. Further, the results of the student evaluations suggest that traditional VM was preferred by learners.

Despite the strong literature base for the use of VM $[3,9,18,19]$, we are unaware of any previous studies investigating its interplay with cognitive load. Early investigations of immersive video technologies [11-15] suggest that they are acceptable to learners and can be an effective educational tool [20]. Building on recent literature suggesting that immersive VM initially increases procedural cognitive load but that this cognitive load decreases with time [15], we hypothesized that incorporating immersive VM at the beginning of procedural training could decrease cognitive load during successive attempts at the procedure. However, our study suggests that this did not occur between sessions or the two groups. There are multiple potential reasons for this: the testing environment may have been persistently stressful, the introduction of a new VM technology may have resulted in an increased extraneous load that further complicated skill acquisition [21], or the three three-hour sessions may not have provided enough time to develop the complex task schema required for CVL insertion, thereby influencing cognitive load and working memory.

Previous research demonstrated increasing procedural competence over the course of multi-session CVC training programs [19-21]. Our findings of improving entrustment scores from the first to last session reflect this, but we did not find a significant difference between the two groups. There could be several reasons for this. Firstly, the concurrent feedback provided in both sessions allowed for the opportunity to facilitate deliberate practice, a method of teaching that depends upon focused, repetitive practice of skill improvement, with feedback [22,23]. The quality of our in-task, concurrent feedback may have overshadowed any positive effect immersive VM could have had on the scoring. This has been recently studied in novice medical students [24], wherein early procedural learning benefitted from VM, but in later procedural interventions students subjectively benefitted more from concurrent feedback. Secondly, we identified that the cognitive load remained higher in the immersive VM group, which may have adversely affected performance. Thirdly, the benefit of VM in the theoretical model of deliberate practice may be rooted in the learners' ability to observe and analyze the performance of experts at key decision points, with expert guidance [23]. Entrustment scores in simulated environments may be an indicator of competence in the clinical setting [25], suggesting that the improvements seen in this workshop may translate to enhanced 
patient care [26]. Procedural time is also a recognized surrogate of procedural mastery, with the level of experience being inversely related to time [21,25-27]. However, we did not find this association in our study. This may have related to the heterogeneity of specialty programs participating in the study or the amount of time (four weeks) between the three training sessions.

Comparisons of the course evaluations between the control and intervention group raise additional concerns regarding the viability of immersive VM for procedural training. While support was available during the sessions for the residents, the results demonstrate significantly lower ratings for the immersive VM group. While statistically significant, this may not reflect a clinically relevant significance to learners and requires a more in-depth assessment as to the reasons behind these differences. Nevertheless, this suggests that despite the initial enthusiasm for this technology from learners [11-15], in the time frame and workshop format provided in this study, it may not have achieved the ease of use or familiarity to learners.

There were limitations to our study. First, we intentionally sought to conduct a pragmatic study by utilizing commercially available equipment. While we achieved this objective, it is possible that more advanced recordings and VM could have been incorporated with additional funds and equipment that may have resulted in an improved learner experience. Second, there were user-onboarding difficulties in the use of the commercial virtual reality (VR) headsets for the learners and instructors. Despite being a commercial product designed for recreational use, there were limitations related to internet connection speeds, user-interface challenges, and navigation within the VR software. As learners and instructors became familiar with the hardware, this became less of a barrier. Third, there was a delay in the completion of the NASA TLX questionnaire by a small minority of participants despite the time being provided for this during the session. This may have subjected these results to recall bias. Finally, 28\% of the participants had previous formal training with CVC insertion. These were evenly distributed between groups, however, this study did not specifically investigate the extent of this formal training, and thus may have influenced the outcomes of interest.

Our study did not demonstrate the benefit of immersive VM over traditional VM, however, there will continue to be opportunities for further investigation in this field. In the short-term, it may be worth investigating the impact of immersive VM for teaching more complex procedures (e.g., intraoperatively) than ultrasound-guided CVC insertion as it may have a larger benefit in these contexts. In the long-term, the permeation of immersive experiences and technologies may become more commonplace, decreasing their cognitive load. Immersive technologies and augmented reality technologies will continue to rapidly evolve and become more intuitive. It will be important to continue to investigate the use of this and newer technology for medical procedural training as it becomes available.

\section{Conclusions}

Simulation using task trainers and video modeling is an effective way to teach medical procedures associated with iatrogenic complications in a low-risk environment. Increasingly sophisticated forms of video modeling have recently become readily available. We hypothesized that teaching ultrasound-guided CVC insertion using immersive VM technology would provide benefits over traditional video modeling. However, the use of immersive VM did not change the cognitive load or improve outcomes over traditional VM when used to augment simulation training for novice resident physicians learning this procedure.

\section{Appendices \\ Appendix A}




\section{Cureus}

Demographic information for RaPID Course - CVC Insertion Study

Thank you for participating in our study

\section{PARTICIPANT INFORMATION AND CONSENT FORM}

STUDY TITLE: Comparing the efficacy of three-dimensional versus two-dimensional video modelling in achieving central venous catheterization competency

\section{PRINCIPLE INVESTIGATOR:}

Dr. Brent Thoma MD, MA, MSc, FRCPC

Department of Emergency Medicine, University of Saskatchewan

Royal University Hospital

103 Hospital Dr.

Saskatoon, SK

Ph. (306)-881-0112

CO-INVESTIGATORS:

Dr. Megan Deck, Anesthesiology Resident, University of Saskatchewan

Dr. Julie Yu, Anesthesiology Resident, University of Saskatchewan

Dr. Joann Kawchuck, Anesthesiologist \& Intensive Care Physician, University of Saskatchewan

Dr. Luke Terrett, Internal Medicine \& Intensive Care Physician, University of Saskatchewan

Dr. Brian Brownbridge, Anesthesiologist \& Intensive Care Physician, University of Saskatchewan

Dr. Kish Lyster, Medical Director for Dilawri Simulation Center, Regina General Hospital, Saskatchewan Health Authority

STUDENT RESEARCHER:

Evan Mah, Medical Student, University of Saskatchewan

\section{INTRODUCTION}

You are invited to take part in this research study because you are a PGY1 resident enrolled in the Resuscitation and Procedural InterDisciplinary (RaPID) curriculum at the University of Saskatchewan. Central venous catheter placement is a common resuscitative procedure. Numerous studies have validated the use of simulation training to practice $\mathrm{CVC}$ insertion, but the most effective method of instruction remain uncertain. The purpose of this study is to compare the quality of procedural performance and the mean time required for residents to successfully perform central venous catheterization (CVC) between participants assigned to either standard two-dimensional video modelling or three-dimensional video capture.

Participation in the RaPID program is required as part of your residency program and will include all aspects of the research study. However, your participation in the research related to this course is voluntary. It is up to you to decide whether or not you wish to take part. If you wish to participate, please indicate this at the bottom of this page. If you do decide to take part in this study, you are still free to withdraw at any time and without giving any reasons for your decision.

\section{FIGURE 5: Appendix A-1}




\section{Cureus}

If you do not wish to participate, you will not lose any benefits and will still participate in all aspects of the curriculum. It will not affect your relationship with any of the researchers or instructors or future evaluation in other medical education opportunities.

Please take time to read the following information carefully. You can ask the researcher to explain any words or information that you do not clearly understand. You may ask as many questions as you need. Please feel free to discuss this with your family, friends or family physician before you decide.

\section{WHY IS THIS STUDY BEING DONE?}

This study is being done to compare the quality of procedural performance and the mean time required for residents to successfully perform central venous catheterization (CVC) between participants assigned to either standard two-dimensional video modelling or three-dimensional video capture.

WHO CAN PARTICIPATE IN THE STUDY?

You are eligible to participate in this study if you are a PGY1 resident enrolled in the Resuscitation and Procedural InterDisciplinary (RaPID) curriculum at the University of Saskatchewan.

WHAT DOES THE STUDY INVOLVE?

Participation in the study will not require any action beyond that required to participate in the CVC module of the RaPID curriculum. The study will randomize you into either the two-dimensional or three-dimensional instructional videos for the procedure, but the course will otherwise be unchanged. It will involve instruction on the insertion of CVC, practice inserting CVC into task trainers using ultrasound, and three recorded assessments of the procedure being performed.

Study data will be collected via:

-A brief questionnaire that will follow this consent form to gather data on the participants' demographics and previous CVC insertion experience.

-A brief survey following each session that will describe your feelings regarding $\mathrm{CVC}$ insertion.

-A program evaluation survey completed at the end of the course.

Those electing not to participate in the research will provide the same data as part of the course, but it will not be used for research purposes.

All research participants will have their videos uploaded to an encrypted, password protected hard drive. After uploading, all copies of these videos will be deleted from the mobile device. The videos will then be reviewed by two or more expert clinicians.

WHAT ARE THE BENEFITS OF PARTICIPATING IN THIS STUDY?

If you choose to participate in this study, there may or may not be direct benefits to you. It is hoped the information gained from this study can be used in the future to benefit other residents learning CVC.

ARE THERE POSSIBLE RISKS AND DISCOMFORTS?

Risks associated with video recording the performance of skills include risks to privacy and confidentiality, including the fear of being thought of poorly from colleagues, peers, or instructors for poor performance. We ask that you do not discuss the performance or other outcomes of other participants during, or after this study for the purposes of confidentiality. We do not anticipate any physical harm or discomfort, but it is possible that participants randomized to the 3D group may become disoriented by the experience. Participants that

FIGURE 6: Appendix A - 2 


\section{Cureus}

do not wish to continue with the study for this reason will be given the chance to watch the video in $2 \mathrm{D}$ instead.

WHAT HAPPENS IF I DECIDE TO WITHDRAW?

While participation in the RaPID CVL module is part of your residency, your participation in this research is voluntary. You may withdraw from this study at any time without providing a reason. There will be no penalty or loss of benefits if you choose to withdraw and your future clinical training or educational standing will not be affected. If you choose to enter the study and then decide to withdraw later. data collected about you that has not already been anonymized for analysis will be removed.

WILL I BE INFORMED OF THE RESULTS OF THE STUDY?

We anticipate that this study will be presented at academic conferences and published in a scholarly journal.

WHAT WILL THE STUDY COST ME?

You will not be charged for any research-related procedures. You will not receive any compensation, or financial benefits for being in this study, or as a result of data obtained from research conducted under this study.

WHAT HAPPENS IF SOMETHING GOES WRONG?

By signing this document, you do not waive any of your legal rights.

WILL MY TAKING PART IN THIS STUDY BE KEPT CONFIDENTIAL?

Your confidentiality will be respected. No information that discloses your identity will be released or published without your specific consent to the disclosure. However, research records identifying you may be inspected in the presence of the Investigator or his or her designate by the University of Saskatchewan Behavioral Research Ethics Board for the purpose of monitoring the research. The results of this study may be presented in a scientific meeting or published, but your identity will not be disclosed

WHO DO I CONTACT IF I HAVE QUESTIONS ABOUT THE STUDY?

If you have any questions or desire further information about this study before or during participation, you can contact Dr. Brent Thoma at 306-881-0112, Dr. Megan Deck at 306-531-2331, or Dr. Julie Yu at 306-655-1183.

If you have any concerns about your rights as a research participant and/or your experiences while participating in this study, contact the Chair of the University of Saskatchewan Research Ethics Board, at 306-966-2975 (out of town calls 1-888-966-2975). The Research Ethics Board is a group of individuals (scientists, physicians, ethicists, lawyers and members of the community) that provide an independent review of human research studies. This study has been reviewed and approved on ethical grounds by the University of Saskatchewan Research Ethics Board.

\section{CONSENT TO PARTICIPATE}

Study Title: Does video feedback and modelling reduce mean time to perform peripheral intravenous cannulation? A pilot study

- I have read (or someone has read to me) the information in this consent form.

- I understand the purpose and procedures and the possible risks and benefits of the study.

- I was given sufficient time to think about it.

- I had the opportunity to ask questions and have received satisfactory answers.

- I understand that I am free to withdraw from this study at any time for any reason and the decision to stop taking part will not

affect my future relationships.

- I give permission to the use and disclosure of my de-identified information collected for the research purposes described in this form.

- I understand that by signing this document I do not waive any of my legal rights.

- I will be given a copy of this consent form (paper or electronic)

1. I agree to participate in this study:

Yes

No

FIGURE 7: Appendix A - 3 


\section{Cureus}

2. Please provide your name and site of training.

Name

City/Town

3. What is your gender?

Male

Female

Other

Prefer not to respond

4. How old are you?

5. Which is your dominant hand?

Left Hand

Right Hand

Ambidextrous

Other (please specify)

6. What Residency Program are you in?

Anesthesiology

Family Medicine-Anesthesia (PGY3)

Emergency Medicine

Family Medicine-Emergency (PGY3)

General Surgery

Internal Medicine

FIGURE 8: Appendix A - 4 


\section{Cureus}

7. Have you received formal Central Venous Catheter insertion training in the past?

Yes

No

8. If you answered yes to Question 6, please describe the training that you received and the context you received it in:

0

25

$50+$

Note the following questions are only for research purposes only, and will not influence your assessment in the RaPID course or your involvement in the study.

10. Did you review the Pre-Course material?

Yes

Partially

FIGURE 9: Appendix A - 5

\section{Appendix B}

Item

360fly 4 K Camera

Samsung S6 Smart Phone

3D Virtual Reality Headset (Samsung Gear VR Reality Headset 2016 Edition)

360 Camera Mount

External Hard Drive (Seagate Backup Plus Slim 1TB)

Samsung S6 Camera Mount

Adobe Video Editing Software (Adobe Premiere Pro)

CVC Insertion Kits

CVC Femoral Task Trainers

CVC Jugular Task Trainers

CVC Femoral Task Trainer Inserts

CVC Jugular Task Trainer Inserts

\begin{tabular}{l|l|l|} 
Vender & Quantity & Total Cost (CAD) \\
\hline Amazon & 1 & $\$ 469.96$ \\
Amazon & 4 & $\$ 2,152.76$ \\
Amazon & 4 & $\$ 259.96$ \\
Amazon & 1 & $\$ 168.99$ \\
Amazon & 1 & $\$ 79.96$ \\
Amazon & 4 & $\$ 107.20$ \\
Adobe & 1 & $\$ 239.98$ (USD) \\
Teleflex & 151 & $\$ 10,570$ \\
Simulab & 2 & $\$ 6,600$ \\
Simulab & 2 & $\$ 5,400$ \\
Simulab & 6 & $\$ 10,200$ \\
Simulab & 6 & $\$ 7,800$
\end{tabular}

TABLE 3: Itemized list of utilized program equipment

\section{Appendix C}




\section{Cureus}

1. Participant Name

The following assessment is used to measure your personal opinion on how much workload was required of you during the task you just completed.

In this assessment, you will first be asked to rate six workload measures.

After you have completed the ratings, you will be asked to compare which of two workload measures is more important than the other when considering the task you just completed. You will be asked to answer 15 of these pairings.

There is no right or wrong answer

2. Mental Demand: How mentally demanding was the task?

Very Low

Very High

3. Physical Demand: How physically demanding was the task?

Very Low

Very High

4. Temporal Demand: How hurried or rushed was the pace of the task?

Very Low

Very High

5. Performance: How successful were you in accomplishing what you were asked to do? Very Low

Very High

6. Effort: How hard did you have to work to accomplish your level of performance? Very Low

FIGURE 10: Appendix C - 1

7. Frustration: How insecure, discouraged, irritated, stressed, and annoyed were you?

Very Low

Very High

FIGURE 11: Appendix C - 2 


\section{Cureus}

Sharek, D. (2009). NASA-TLX Online Tool (Version 0.06)[internet Application]. Research Triangle, NC. Retrieved from http://mww.nasatll.com

8. Of the two workload measures below, which one contributed the most to the task you just completed? Frustration

Effort

9. Of the two workload measures below, which one contributed the most to the task you just completed? Mental Demand

Effort

10. Of the two workload measures below, which one contributed the most to the task you just completed? Effort

Performance

11. Of the two workload measures below, which one contributed the most to the task you just completed? Performance

Temporal Demand

12. Of the two workload measures below, which one contributed the most to the task you just completed? Temporal Demand

Frustration

13. Of the two workload measures below, which one contributed the most to the task you just completed? Temporal Demand

Effort

14. Of the two workload measures below, which one contributed the most to the task you just completed? Temporal Demand

Mental Demand

FIGURE 12: Appendix C - 3 


\section{Cureus}

15. Of the two workload measures below, which one contributed the most to the task you just completed? Effort

Physical Demand

16. Of the two workload measures below, which one contributed the most to the task you just completed? Physical Deamdn

Temporal Demand

17. Of the two workload measures below, which one contributed the most to the task you just completed? Performance

Mental Demand

18. Of the two workload measures below, which one contributed the most to the task you just completed? Frustration

Mental Demand

19. Of the two workload measures below, which one contributed the most to the task you just completed? Mental Demand

Physical Demand

20. Of the two workload measures below, which one contributed the most to the task you just completed? Physical Demand

Performance

21. Of the two workload measures below, which one contributed the most to the task you just completed? Physical Demand

Frustration

22. Of the two workload measures below, which one contributed the most to the task you just completed? Performance

Frustration

FIGURE 13: Appendix C - 4

\section{Appendix D}




\section{Cureus}

1. Evaluator Name

2. Participant ID

3. Video Number

Based on the video, please evaluate the residents entrustability to complete the following Entrustable Professional Activity:

4. The resident is able to place a central venous catheter under ultrasound guidance with appropriate preparation, draping, and confirmation of venous placement.
1 - "I had to do"
4 - "I needed to be there just in case"
2 - "I had to talk them through"
5 - "I didn't need to be there
3 - "I needed to prompt"

Please rank the extent the participant was able to perform these specific technical abilities

5. Time and Motion

Not competent to perform independently. Many unnecessary movements

Competent to perform
independently.
movements.
Above average to perform independently.

Economy of movement and maximum efficiency.

6. Instrument Handling

Not competent to perform independently.

Repeated awkward or tentative Borderline competent to perform handling of instruments. independently.

Competent to perform
independently.
Competent, occasionally
appeared stiff or awkward.

Above average to perform independently. Fluid handling of instruments, no stiffness or awkwardness.

FIGURE 14: Appendix D - 1

7. Knowledge of Instruments Not competent to perform independently. instruments. independently.

8. Flow of Procedure

Not competent to perform independently.

Frequently stopped and seemed Borderline competent to perform unsure of next step. independently.

\section{Competent to perform independently. \\ Demonstrated reasonable progression of procedure.}

\section{Above average to perform independently. \\ Obviously planned procedure, smooth flow.}

\section{Knowledge of Specific Procedure}

Not competent to perform independently.

Competent to perform independently.

Above average to perform independently. Would have needed instruction Borderline competent to perform Seemed to know most steps of Demonstrated familiarity with all
to complete most steps.
procedure.
aspects of procedure.

FIGURE 15: Appendix D - 2 


\section{Cureus}

10. Did the participant display the following?

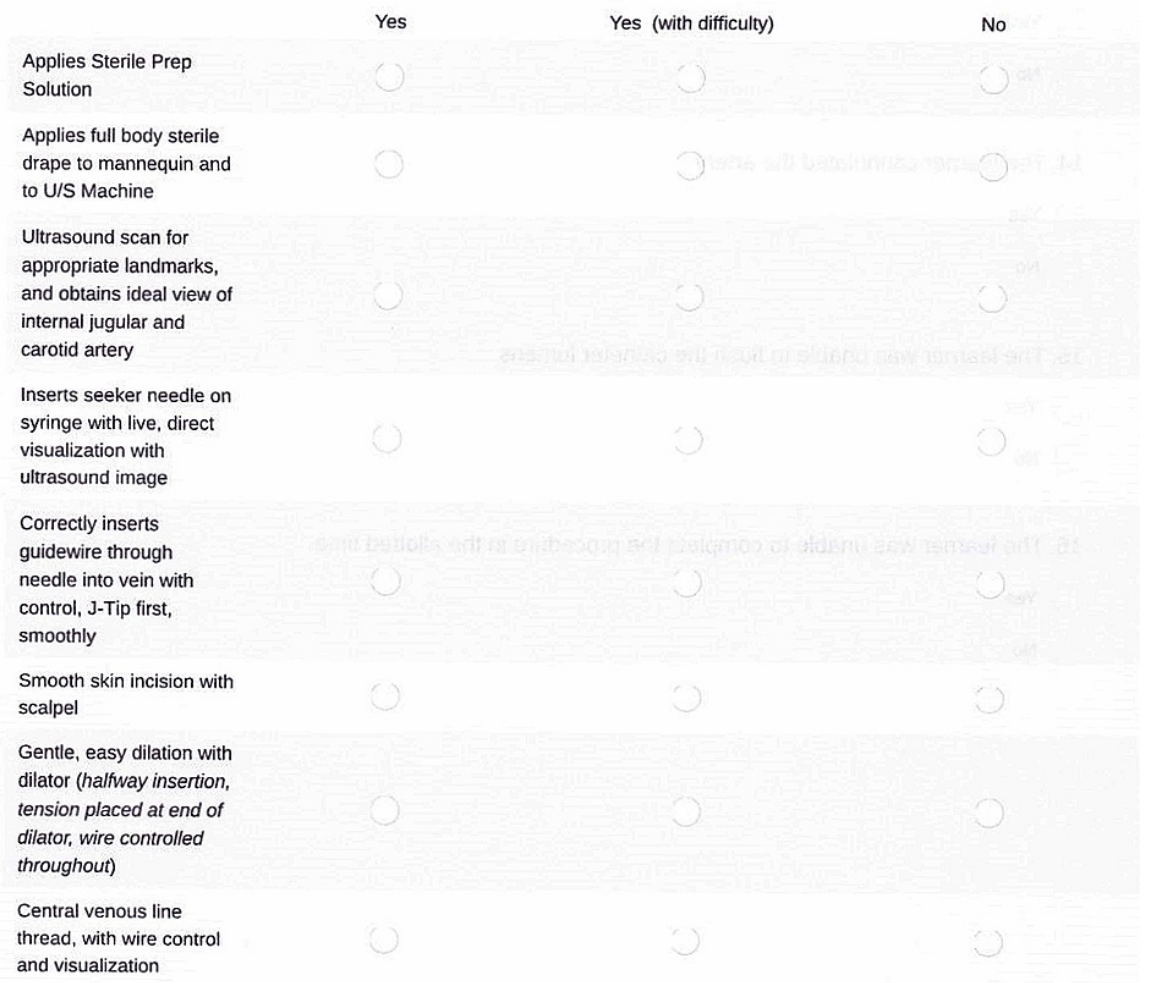

11. How many attempts were made

Did any of the following occur?

12. The learner contaminated the sterile field

Yes

No

FIGURE 16: Appendix D - 3 


\section{Cureus}

13. The learner punctured the artery

Yes

No

14. The learner cannulated the artery

Yes

No

15. The learner was unable to flush the catheter lumens

Yes

No

16. The learner was unable to complete the procedure in the allotted time

Yes

No

FIGURE 17: Appendix D - 4

\section{Appendix E}

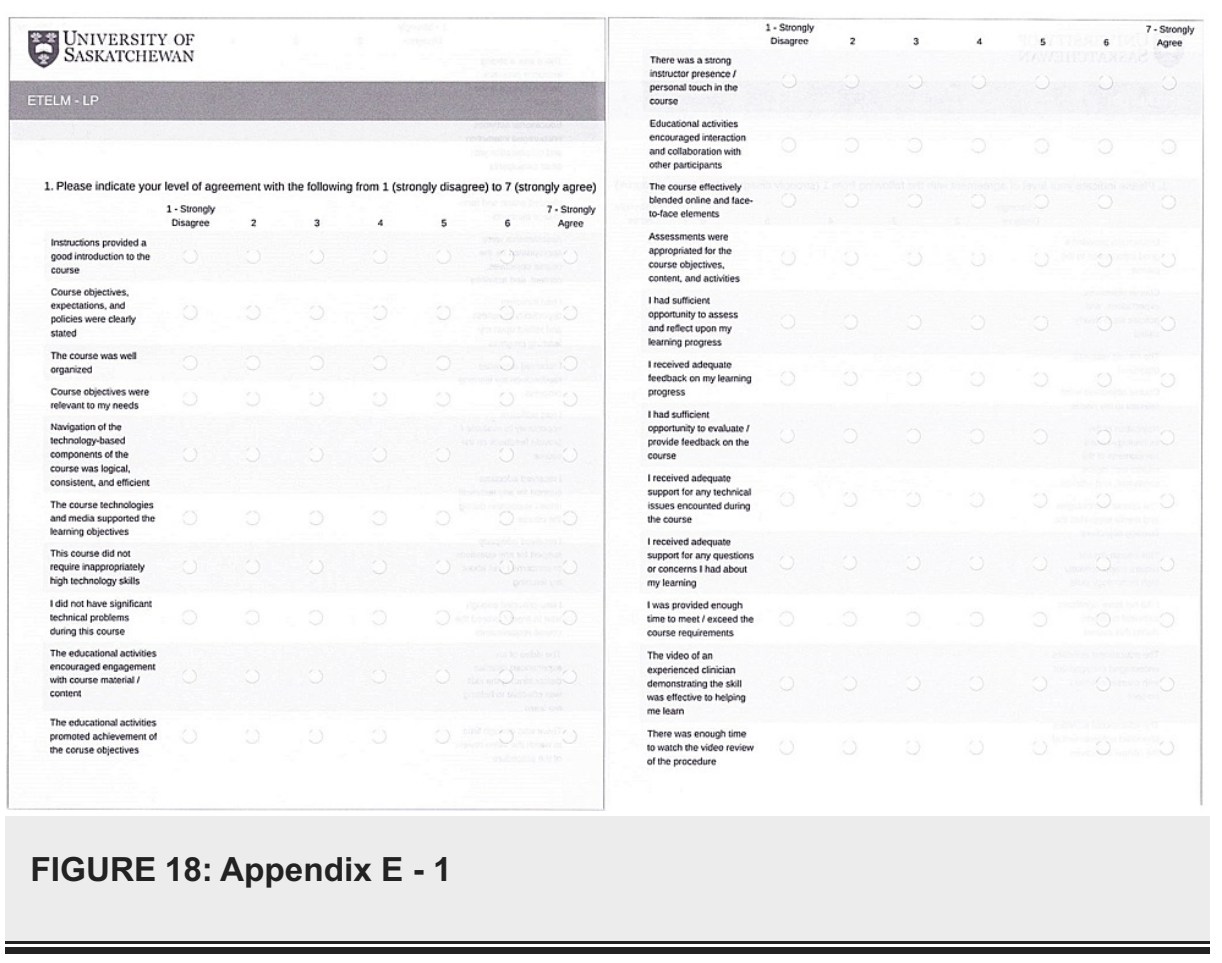




\section{Cureus}

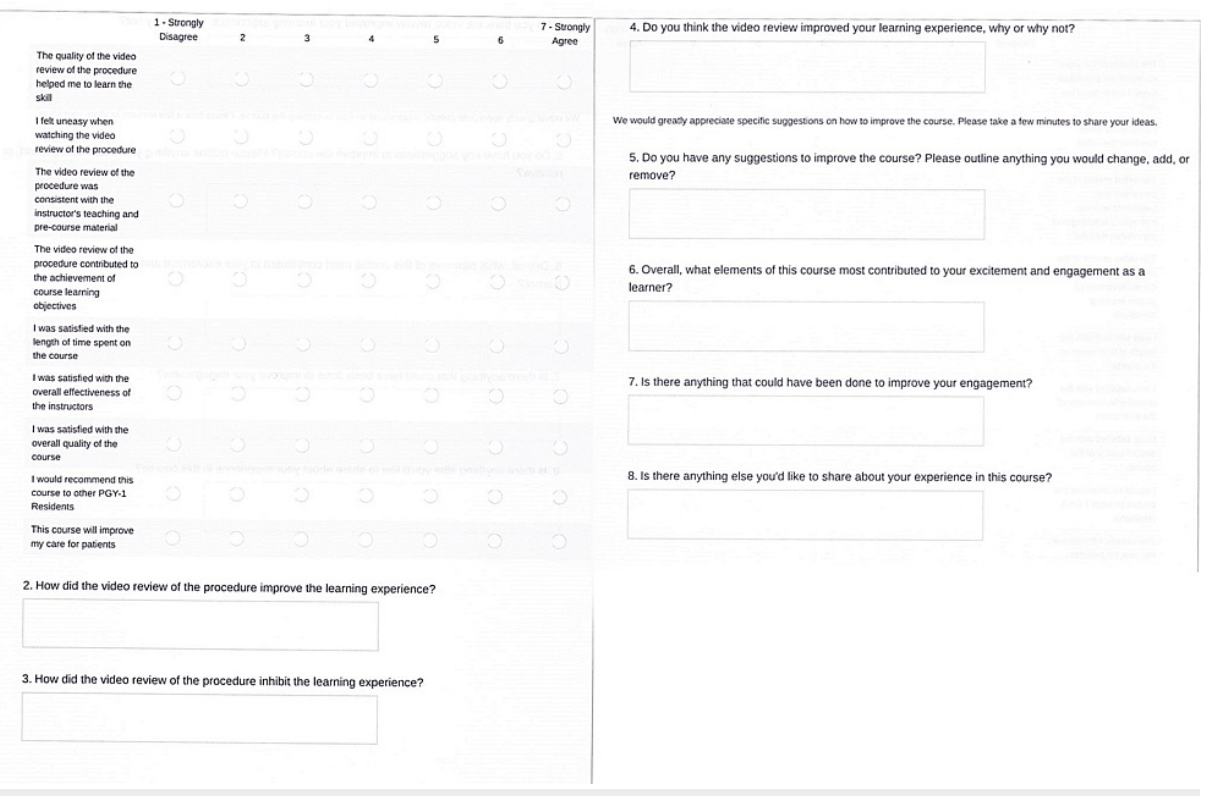

FIGURE 19: Appendix E - 2

\section{Appendix F}




\section{Cureus}

\section{ETELM Descriptor}

Instructions provided a good introduction to the course

Course objectives, expectations, and policies were clearly stated

The course was well organized

Course objectives were relevant to my needs

Navigation of the technology-based components of the course was logical, consistent, and efficient

The course technologies and media supported the learning objectives

This course did not require inappropriately high technology skills

I did not have significant technical problems during this course

The educational activities encouraged engagement with course material / content

The educational activities promoted achievement of the course objectives

There was a strong instructor presence / personal touch in the course

Educational activities encouraged interaction and collaboration with other participants

The course effectively blended online and face-to-face elements

Assessments were appropriate for the course objectives, content, and activities

I had sufficient opportunity to assess and reflect upon my learning progress

I received adequate feedback on my learning progress

I had sufficient opportunity to evaluate / provide feedback on the course

I received adequate support for any technical issues encountered during the course

I received adequate support for any questions or concerns I had about my learning

I was provided enough time to meet / exceed the course requirements

The video of an experienced clinician demonstrating the skill was effective to helping me learn

There was enough time to watch the video review of the procedure

The quality of the video review of the procedure helped me to learn the skill

I felt uneasy when watching the video review of the procedure

The video review of the procedure was consistent with the instructor's teaching and pre-course material

The video review of the procedure contributed to the achievement of course learning objectives

$$
5.9
$$

I was satisfied with the length of time spent on the course

TABLE 4: Modified evaluation of technology-enhanced learning materials - learner perspective (ETELM-LP) results

1: Strongly Disagree; 2: Disagree; 3: Somewhat Disagree; 4: Neither Agree Nor Disagree; 5: Somewhat Agree; 6: Agree; 7: Strongly Agree

\section{Additional Information}

\section{Disclosures}


Human subjects: Consent was obtained or waived by all participants in this study. University of Saskatchewan Research Ethics Board issued approval BIO\#18-46. In the opinion of the Research Ethics Board (REB) this submission is exempt from the requirement of the Research Ethics Board (REB) review and approval based on article 2.5 of the Tri-Council Policy Statement (TCPS2). Article 2.5 specifies "quality assurance and quality improvement studies, program evaluation activities, and performance reviews, or testing with normal educational requirements when used exclusively for assessment, management or improvement purposes do not constitute research for the purposes of this Policy, and do not fall within the scope of REB review". . Animal subjects: All authors have confirmed that this study did not involve animal subjects or tissue. Conflicts of interest: In compliance with the ICMJE uniform disclosure form, all authors declare the following: Payment/services info: This work was supported by the University of Saskatchewan College of Medicine for program fees, as well as the Royal University Hospital Foundation (Saskatoon, Canada) Helping-Understanding-Giving (HUG) Grant, and the University of Saskatchewan's College of Medicine Dean's Summer Research Award. The authors have no other financial declarations. Financial relationships: All authors have declared that they have no financial relationships at present or within the previous three years with any organizations that might have an interest in the submitted work. Other relationships: All authors have declared that there are no other relationships or activities that could appear to have influenced the submitted work.

\section{Acknowledgements}

We would like to acknowledge the contributions of Drs. Brian Brownbridge and Luke Terrett to this research.

\section{References}

1. Velasquez Reyes DC, Bloomer M, Morphet J: Prevention of central venous line associated bloodstream infections in adult intensive care units: a systematic review. Intensive Crit Care Nurs. 2017, 43:12-22. 10.1016/j.iccn.2017.05.006

2. Polderman KH, Girbes AR: Central venous catheter use part 1: mechanical complications . Intensive Care Med. 2002, 28:1-17. 10.1007/s00134-001-1154-9

3. Boyer E, Miltenberger RG, Batsche C, Fogel V: Video modeling by experts with video feedback to enhance gymnastics skills. J Appl Behav Anal. 2009, 42:855-860. 10.1901/jaba.2009.42-855

4. Ram N, Riggs SM, Skaling S, Landers DM, McCullagh P: A comparison of modelling and imagery in the acquisition and retention of motor skills. J Sports Sci. 2007, 25:587-597. 10.1080/02640410600947132

5. Kunkler K: The role of medical simulation: an overview. Int J Med Robot Comput Assist Surg. 2006, 2:203210. 10.1002/rcs.101

6. Corvetto MA, Pedemonte JC, Varas D, Fuentes C, Altermatt FR: Simulation-based training program with deliberate practice for ultrasound-guided jugular central venous catheter placement. Acta Anaesthesiol Scand. 2017, 61:1184-1191. 10.1111/aas.12937

7. Barsuk JH, McGaghie WC, Cohen ER, O’Leary KJ, Wayne DB: Simulation-based mastery learning reduces complications during central venous catheter insertion in a medical intensive care unit*. Crit Care Med. 2009, 37:2697-2701. 10.1097/CCM.0b013e3181a57bc1

8. Zetou E, Tzetzis G, Vernadakis N, Kioumourtzoglou E: Modeling in learning two volleyball skills. Percept Mot Skills. 2002, 94:1131-1142. 10.2466/pms.2002.94.3c.1131

9. Fukkink RG, Trienekens N, Kramer LJC: Video feedback in education and training: putting learning in the picture. Educ Psychol Rev. 2011, 23:45-63. 10.1007/s10648-010-9144-5

10. Saleh GM, Wawrzynski JR, Saha K, et al.: Feasibility of the human factors immersive simulation training in opthalmology. JAMA Opthalmol. 2016, 134:905-911. 10.1001/jamaophthalmol.2016.1769

11. Huber T, Wunderling T, Paschold M, Lang H, Kneist W, Hansen C: Highly immersive virtual reality laparoscopy simulation: development and future aspects. Int J Comput Assist Radiol Surg. 2018, 13:281-290. 10.1007/s11548-017-1686-2

12. Huber T, Paschold M, Hansen C, Wunderling T, Lang H, Kneist W: New dimensions in surgical training: immersive virtual reality laproscopic simulation exhilarates surgical staff. Surg Endosc. 2017, 31:4472-4477. 10.1007/s00464-017-5500-6

13. Herault RC, Lincke A, Milrad M, Forsgärde E-S, Elmqvist C: Using 360-degrees interactive videos in patient trauma treatment education: design, development and evaluation aspects. Smart Learn Environ. 2018, 5:26. 10.1186/s40561-018-0074-X

14. Pulijala Y, Ma M, Pears M, Peebles D, Ayoub A: Effectiveness of immersive virtual reality in surgical training -a randomized control trial. J Oral Maxillofac Surg. 2018, 76:1065-1072. 10.1016/j.joms.2017.10.002

15. Frederiksen JG, Sørensen SMD, Konge L, et al.: Cognitive load and performance in immersive virtual reality versus conventional virtual reality simulation training of laparoscopic surgery: a randomized trial. Surg Endosc. 2020, 34:1244-1252. 10.1007/s00464-019-06887-8

16. Gofton WT, Dudek NL, Wood TJ, Balaa F, Hamstra SJ: The Ottawa Surgical Competency Operating Room Evaluation (O-SCORE): a tool to assess surgical competence. Acad Med. 2012, 87:1401-1407. 10.1097/ACM.0b013e3182677805

17. Cook DA, Ellaway RH: Evaluating technology-enhanced learning: a comprehensive framework. Med Teach. 2015, 37:961-970. 10.3109/0142159X.2015.1009024

18. Obrusnikova I, Rattigan PJ: Using video-based modeling to promote acquisition of fundamental motor skills. J Phys Educ Recreat Danc. 2016, 87:24-29. 10.1080/07303084.2016.1141728

19. Stefanidis D, Korndorffer JR, Heniford BT, Scott DJ: Limited feedback and video tutorials optimize learning and resource utilization during laparoscopic simulator training. Surgery. 2007, 142:202-206. 10.1016/j.surg.2007.03.009

20. Kirkpatrick D: Revisiting Kirkpatrick's four-level model. Train Dev. 1996, 50:54-57.

21. Andersen SAW, Mikkelsen PT, Konge L, Cayé-Thomasen P, Sørensen MS: The effect of implementing 


\section{Cureus}

cognitive load theory-based design principles in virtual reality simulation training of surgical skills: a randomized controlled trial. Adv Simul. 2016, 1:20. 10.1186/s41077-016-0022-1

22. Erickson K, Anders RT, Tescher-Romer C: The role of deliberate practice in the acquisition of expert performance. Psychol Rev. 1993, 100:363-406.

23. Ericsson KA: Necessity is the mother of invention: video recording firsthand perspectives of critical medical procedures to make simulated training more effective. Acad Med. 2014, 89:17-20. 10.1097/ACM.0000000000000049

24. Yu J, Lo C, Madampage C, et al.: Video modeling and video feedback to reduce time to perform intravenous cannulation in medical students: a randomized-controlled mixed-methods study. Can J Anesth. 2020, 67:715-725. 10.1007/s12630-020-01570-2

25. Weersink K, Hall AK, Rich J, Szulewski A, Dagnone JD: Simulation versus real-world performance: a direct comparison of emergency medicine resident resuscitation entrustment scoring. Adv Simul. 2019, 4:9. 10.1186/s41077-019-0099-4

26. McGraw R, Chaplin T, McKaigney C, et al.: Development and evaluation of a simulation-based curriculum for ultrasound-guided central venous catheterization. Can J Emerg Med. 2016, 18:405-413.

10.1017/cem.2016.329

27. Clinkard D, Holden M, Ungi T, Messenger D, Davison C, Fichtinger G, McGraw R: The development and validation of hand motion analysis to evaluate competency in central line catheterization. Acad Emerg Med. 2015, 22:212-218. 10.1111/acem.12590 